\title{
Meaningful Gameplay Design and the Effect on Eudaimonic and Hedonic Gaming Experience
}

\author{
Wei Jie Dominic Koek \\ Graduate College, \\ Nanyang Technological University \\ koek0002@e.ntu.edu.sg
}

\author{
Vivian Hsueh Hua Chen \\ Wee Kim Wee School of \\ Communication and Information, \\ Nanyang Technological University \\ chenhh@ntu.edu.sg
}

\author{
Valerie $\mathrm{Yu}$ \\ Wee Kim Wee School of \\ Communication and Information, \\ Nanyang Technological University \\ valeriej001@e.ntu.edu.sg
}

\begin{abstract}
Past research has explored meaningfulness in video game narratives, as well as meaningful play. However, more empirical studies are needed to understand the mechanisms of meaningful play. This paper first investigates how the design of game dialogues may enable meaningfulness in video games (eudaimonic gaming experience) and how it relates to game enjoyment (hedonic gaming experience). Second, it also examines how meaningful play influences prosocial attitudes toward culturally different outgroups. A pre-posttest experiment of a management game was conducted with 174 adult participants. Participants were randomly assigned to either a game with moral scenarios or one without. Results showed that participants who played the game with moral decision-making perceived a higher level of meaningfulness and game enjoyment. No significant relationship was found between perceived meaningfulness and intergroup perceptions.
\end{abstract}

\section{Introduction}

Video games are increasingly being used for serious purposes beyond entertainment. From technical simulations to exergames to persuasion, these deviations from traditional entertainment video games have become popularized as forms of meaningful play. Meaningful play holds varying definitions across these different use cases and have been implemented using differing features of video games. In some cases, meaningful play in video games can be defined as an emergent experience from playersystem interaction that results in outcomes that are both discernible and relevant to the context of a game [1]. Other existing evidence suggests that game experiences can transcend game boundaries in the form of transformative experiences beyond the context of the game [2]. Evidence of such transformative experiences, for example, has been observed in various implementations of persuasive games in the form of attitude and behavior change following gameplay [3, 4, 5]. A study conducted by Iten et al. [4] on whether prosocial decisions in video games could also lead to increased prosocial behavior in real life found that choosing to help a character in a video game affected their subsequent decision to help in real-life by donating to a charity. A series of studies conducted by Gentile et al. [5] also produced evidence that prosocial gameplay was positively related to prosocial behaviors and traits such as helping, cooperation and sharing, empathy, and emotional awareness. The authors additionally note that while there are various dimensions in which video games can have effects (e.g., context, structure, mechanics), most existing studies emphasize on game content as its framing and exposure to players can affect and reinforce positive appraisals of such stimuli through cognitive processing of the content [5].

Despite knowing that game content can facilitate subsequent positive attitudes and behaviors, challenges remain in relation to considerations in designing a video game that aims to achieve these outcomes. The first of which is the question of how such meaningful content can be designed and integrated effectively in a video game. Second, studies that explore transformation experiences in video gameplay are based on theoretically driven processes but lack an elaboration of how underlying gameplay mechanisms may drive or enhance these processes. Theoretically, meaningful gameplay is often associated with eudaimonic gaming experience. Fun gameplay is linked to hedonic gaming experience. However, it is unclear as to how these two coexist in gaming experience empirically. Hence, this study aims to explore the following research questions through the development of a custom-made prosocial game:

RQ1: How can a prosocial game be designed to be both meaningful and enjoyable? 
RQ2: How does perceived meaningfulness of a prosocial game influence intergroup perceptions?

By investigating how the design of game dialogues may facilitate eudaimonia and hedonia in a video game, this study hopes to shed some light on designing meaningful play through the use of empirical findings. Additionally, this study also seeks to explore how the design of meaningful play in a contextually relevant game may also influence prosocial outcomes toward culturally different outgroups.

\section{Literature Review}

\subsection{Meaningfulness}

In dictionary definitions of meaningfulness, the term is described as having the quality of being useful, serious, or important [6], and synonymous with significance [7]. Some common examples of its application include positive psychology and organizational behavior. In positive psychology, it is described as the multifaceted fulfilment of four basic needs of purpose, values, self-efficacy, and self-worth [8] or alternatively competence, autonomy, relatedness, and beneficence [9]. In the context of organizational behavior, meaningfulness refers to positive associations toward an activity (commonly relating to the workplace) that is seen to be important or fulfilling $[10,11]$. On a preliminary level, despite the different contexts where meaningfulness is situated, they bear similarities in terms of polarity and individual perception, where meaningfulness is a positive experience of something perceived to be beneficial to an individual in some way. More recently, the concept of meaningfulness has also been extended to describe video game experiences in various ways. Laato et al. [2] suggests that identification with video game characters and story supports meaning making within the virtual world; this form of meaningfulness is connected to nostalgia when players relate their game experiences to past experiences and knowledge. Another way meaningfulness can occur in video games is through the perception that playing the video game provides them with tangible benefits (e.g., exercise benefits from playing Pokémon GO) [12]. Meaningfulness in video games can also be attributed to goal fulfilment and need satisfaction experienced by players as a result of accomplishments that are achieved when playing a video game [13].

Meaningfulness, more generally, relates to a form of happiness known as eudaimonia, and while positive affect is often found to be correlated with meaningfulness, they are distinct concepts with positive affect relating more closely to feelings of happiness known as hedonia; more importantly, meaningfulness can still be experienced in situations where positive affect is absent [14]. In distinguishing hedonia from eudaimonia, scholars have proposed that hedonic happiness refers to pleasurable feelings and emotions, while eudaimonic happiness refers to feelings of self-realization and personal development [15]. Entertainment media such as movies and video games are suggested to be sources of both hedonic and eudaimonic gratification [15]. Eudaimonic gratification refers to an audience response that is elicited by the portrayal of thought-provoking entertainment media content (e.g., moral, altruistic), and has also been found to be related to positive sociomotivational effects, such as improvements in prosocial attitudes and behaviors $[16,17,18]$.

Video games are a form of entertainment media that have been used in such ways to promote positive attitudes and behaviors [19], However, the mechanisms by which video gameplay and meaningfulness are linked could benefit from further elaboration. Designing a video game that is meaningful to players could help to promote prosocial outcomes through the process of introspection [20, 21]. Meaningfulness derived from engaging with gameplay has often been anecdotally described by gamers in relation to entertainment game titles, and the ability to replicate such designed experiences may be beneficial in achieving more serious gaming outcomes. In order to interweave meaningfulness into a video game, we also need to explore how video game mechanics can support meaningful play.

\subsection{Meaningful Play}

Within the context of a video game, two primary sources of meaningful play that are frequently discussed include gameplay and narrative. From the gameplay perspective, Salen and Zimmerman [1] propose that meaningful play emerges from in-game interactions that have outcomes that are discernible and integrated, driven by conscious choices players make to play a game. Discernible in this sense means that the outcomes of player action are clearly communicated and visible to players (e.g., by feedback), and integrated means that the outcome of every player action can also be related to the game as a whole [1]. It is important to note that these outcomes in the context of gameplay are not the same as consequences in the context of narrative where it is suggested that consequential choices change the trajectory and directly impact the story (e.g., [22, 23]). Rather, it suggests that meaningfulness can be elicited as a result of mere player-game interaction. From the 
need fulfilment standpoint, this may be the case especially with gameplay choices or actions that allow players to experience a sense of autonomy and competence [24].

In role-playing games, for example, dialogue choices can facilitate interactivity by allowing players to progress through the game at a pace that they dictate while also having a perceived role to play in unraveling the storyline [25]. In these games, a predesigned narrative tends to be a central feature of the game. However, scholars have also proposed an alternative view that meaningfulness can still emerge in games without a clearly prescribed narrative. For example, in open-world or simulation games like Minecraft or The Sims, players are allowed to exhibit their own creativity in playing the game, thus allowing them to derive meaning purely from their own in-game actions and choices [26]. Further evidence for this has been found in studies showing that the presence of interactivity in video games can lead to higher levels of perceived meaningfulness [19].

While decision-making and player action within video games can create meaningful experiences for players, findings from player interviews conducted by Rogers et al. [27] suggest that beyond the mechanism of simply making a choice or carrying out specific ingame actions, meaningfulness may be further enhanced by also considering the content of these choices or actions and how they engage players cognitively. One way that video games can do this is by introducing moral dilemmas into choices that players are asked to make over the course of the game. Moral dilemmas can be understood as situations where multiple moral values or duties have to be considered, but only one can be upheld [28]. According to Green and Jenkins [29], video games provide an avenue for people to interact with the narratives and those interactions might evoke a sense of responsibility among players as they become liable for their in-game actions. Graham and colleagues [30] suggest that the moral reasoning process differs for each individual and is influenced by factors such as prior moral development, cultural differences, and intuition. Hence, it is reasonable to suggest that interweaving moral dilemmas into gameplay would trigger differing cognitive and automatic responses in different players. For example, players might be placed in a position where they contemplate various in-game choices, evaluate if they had made the correct decisions, and consider alternatives to the game's narratives [29]. The presence of moral dilemmas could also divert players' attention to values/principles in video games, if those scenarios are well presented [31].

In the commercial game, Papers, Please, players take on the role of a border inspector who has to check the documents of people intending to cross the border. Players are faced with the dilemma of earning sufficient credits to support their family members, while empathizing with the stories of people they meet at the border. Boltz et al. [32] argue that the game puts players in a position where they would doubt the "procedural rhetoric" (p. 6), and this enables them to engage with the perspective of the character they are playing to question the "underlying ideologies and philosophy" (p. 7) of the game settings. This suggests that in designing for meaningful play, providing players with morally weighted choices that require more conscious thought processing and those that they can relate to may facilitate meaning making more effectively than choices that can be made in a more flippant or arbitrary manner.

H1: Players will perceive greater meaningfulness in a game with moral choices than in the same game without moral choices.

Assuming that meaningfulness in a game fulfils eudaimonic enjoyment for players [19], it is necessary on the other hand to also consider how hedonic enjoyment is experienced in a game, as fun is a central component and consideration for video games [27]. Oliver and Bartsch [16] argue that fun is more often found in non-meaningful games that tend to be shallower in content and cognitive load requirements in comparison to meaningful games and tend to be entertaining in distracting rather than thoughtprovoking ways. Kümpel and Unkel [33], in an experiment comparing the effects of eudaimonic vs. hedonic video game experiences, found that players associated eudaimonic and hedonic video game experiences with different types of need satisfaction, and in relation to different features of the game. For example, eudaimonic experiences were typically associated with the story and perceived cognitive challenge, while hedonic experiences were associated with more favourable evaluations of a game's controls [34]. Supported by Tamborini et al.'s [35] study which found hedonic and non-hedonic needs, while distinct, contributed in a complementary manner to media enjoyment of a video game, this suggests that a video game fulfilling both eudaimonic and hedonic entertainment experiences may lead to greater overall enjoyment of a video game as they address different player needs, and can co-exist in the same game. Hence, a video game designed similarly with differing manipulations for meaningfulness based on game content may lead to differences in players' overall enjoyment as one version would potentially fulfil both hedonic and eudaimonic needs, while the other would only fulfil hedonic needs. Thus, it is predicted that: 
H2: Players who play a moral version of a game would report higher game enjoyment than those who play the neutral (non-moral) version.

\subsection{Meaningful Play and Outgroup Admiration}

Improving players' perceived meaningfulness of games may also be beneficial for serious games that aim to achieve positive outcomes following gameplay. In other forms of entertainment media, it has been observed that by providing opportunities for the audience to contemplate about morally provoking issues, it can elicit feelings of inspiration through the thought-provoking content and encourage individuals to think and act prosocially in real life [27]. An experiment conducted by Steinemann et al. [36] found that players' perceived meaningfulness of video game narrative content was a significant predictor of their subsequent willingness to help in the form of donating behavior. This suggests that meaningfulness may be an important game design consideration for serious games that aim to promote prosocial outcomes following context-specific gameplay.

In the earlier section, it was discussed that morally thought-provoking content could elicit a greater sense of meaning for players in relation to video gameplay. However, the links between perceived meaningfulness and more specific prosocial outcomes warrant further elaboration. A focus group study by Holl et al. [37] exploring players' engagement with moral content in games could lend some preliminary insight into this process. In the study, players explained how moral decisions made them feel more involved with the story, and how it made them reflect more about their decisions in the interactive scenarios. This suggests that situating moral decisions within certain contextspecific social interactions may enhance the way individuals think about such issues and prompt retrospection that influences future decision-making [38, 39]. Disposition theory [40] suggests that when characters are involved in entertainment media narratives, individuals form affective dispositions toward them through a moral judgment process by assessing their actions within the narrative. While this has been traditionally used to understand affective disposition formation in passive media such as television dramas, its mechanisms may be similarly useful for understanding how players may perceive characters in interactive media such as video games.

When presented with moral choices in a video game, players are responsible for the decisions made and hence it is plausible to assume that they would form positive moral judgments and affective dispositions toward the character(s) centrally involved in the moral decision. Admiration is an example of an affective state [41] that could be examined in relation to subsequent prosocial outcomes. According to Sweetman et al. [42], admiration can be defined as one's appraisal toward "legitimate status and/or power." Admiration toward another person or group of people should be related to helping behavior or cooperation with members of that group, based on the stereotype content model (SCM) point-of-view [42].

The parasocial contact hypothesis suggests that individuals may perceive mediated interaction in a similar way to interpersonal interaction, extending the benefits of intergroup contact and facilitating positive social responses to outgroup members involved in the parasocial interaction [43]. In a video game experiment that examined the effects of parasocial contact with outgroup non-player characters, the results suggested that such parasocial contact had the ability to reduce explicit biases and improve outgroup attitudes [44] and drew attention to the possibility of incorporating outgroup members in video game character design to foster positive attitudes.

Exploring how one's admiration toward an outgroup represented by a non-player character changes following engagement with moral content provides a preliminary direction for understanding how meaningful play, in this case driven by moral decision-making, may facilitate positive attitude change toward outgroup members. Hence, we propose that:

H3: Players who play the game with moral choices will have greater admiration change toward cultural outgroups, as compared to those who play the game without moral choices.

H4: Players who perceive the game to be more meaningful will have greater admiration toward cultural outgroups.

\section{Method}

\subsection{Game User Study Design}

A narrative game was designed for this study. The game was designed to be a management game in which players had to run a food establishment as their business and manage resources, including goods, manpower, and services. The hedonic elements of the game were broadly designed around Schell's proposed "Lens of Fun" for video game design [45], capitalizing on player motivation by providing gameplay goals and challenges (e.g., achievements, resource balancing), and using the element of surprise to keep players engaged through quick-time events in the form of mini games and decision-making dialogues. Contextspecific moral dilemmas were then included in the 
decision-making dialogues for the experimental condition to facilitate eudaimonic enjoyment. The ultimate goal of the game was to maintain profits so that players would be able to sell the food court for a high price to another owner eventually. The employees in the game comprised predominantly immigrant workers of lower socioeconomic status or from older age groups who were different from participants in this study. Participants in the study were either citizens or new citizens and came from a higher education background and were of a younger age. Aside from managing supplies, manpower, sales, and staff appraisal, players had to make decisions involving customer-employee relationships and employees' welfare-related issues in the game.

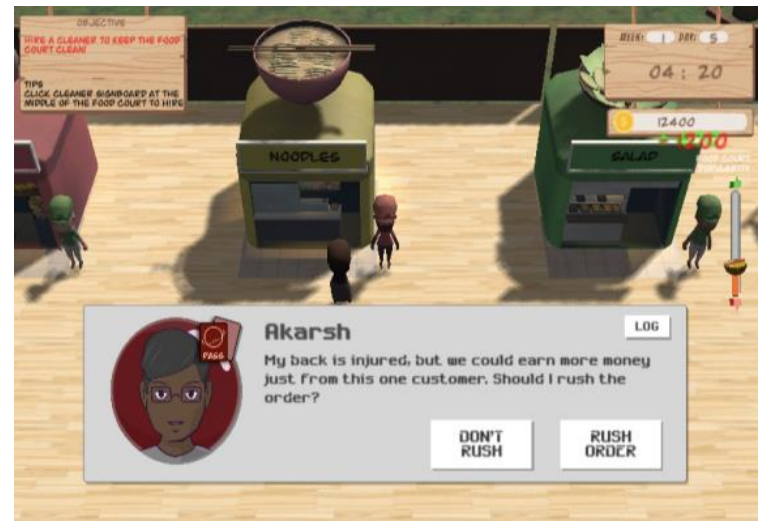

Figure 1. Example of a moral scenario in the game which asked players to decide between rushing or not rushing a worker who is experiencing a back injury

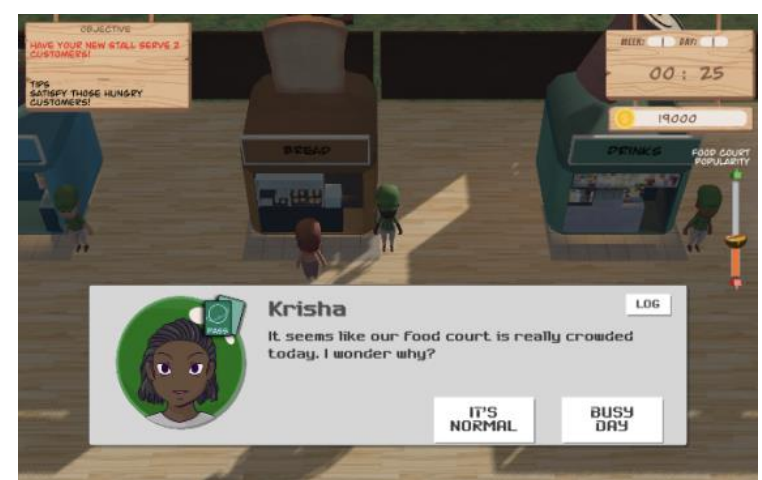

Figure 2. Example of neutral scenario in the game which asked players about their thoughts regarding the food establishment's crowd level

There were two game versions that differed in terms of the decision-making scenarios. Players were randomly assigned to either play the moral or neutral game version. The moral version contained scenarios that required players to decide between helping their workers or supporting the customers, which could either have an impact on the employee's welfare (refer to Figure 1 for example scenario) or the earnings of the food establishment. The neutral version presented scenarios that did not influence the workers' wellbeing, regardless of players' choices (refer to Figure 2 for example scenario). In the moral version, players' decisions would not cause them to ultimately lose the game. The choices would lead to either a decrease in the food establishment's credits or popularity (increase in customers), followed by the increase of the other metric, which would still benefit the establishment's development. In the neutral version, players' decisions did not influence the credits and popularity of the food establishment.

A pre-posttest study design was used to examine how players perceived the meaningfulness of the game and how the game influenced players' feelings of admiration toward cultural outgroups. Participants first completed a pre-game questionnaire which measured their admiration toward cultural outgroups and their demographic information, such as age, gender, and nationality. After three to five days, a link containing the video game was sent to participants through email. Participants played the game and filled out a post-game survey via Qualtrics. Gameplay lasted for approximately 20 to 25 minutes. The post-game questionnaire measured their perceived meaningfulness of the game play, perceptions toward the overall game experience, and admiration toward cultural outgroups.

\subsection{Participants}

A total of 174 participants were recruited through research recruitment channels on the messaging platform Telegram, as well as a university in Singapore. Participants were compensated with either S\$10 gift vouchers or course credits, upon completion of the study. The participants' mean age was 22.86 $(S D=2.80)$, with 110 females $(63.2 \%), 63$ males $(36.2 \%)$ and 1 other $(0.6 \%) .169$ of them were Singapore citizens $(97.1 \%)$ and 5 were permanent residents (2.9\%). Among the participants, 47 of them reported a total household income of $\$ \$ 9,000$ and above (27.0\%), 63 between $\mathrm{S} \$ 3,000$ to $\$ \$ 8,999$ (36.2\%), 27 below S\$3,000 (15.5\%), and 37 who did not know/refused to answer $(21.3 \%)$. In terms of highest education attained, 69 of them completed Alevel $(39.7 \%)$, 50 had a bachelor's degree (28.7\%), 53 of them had a diploma (30.5\%), and 2 others $(1.1 \%)$.

A total of 90 participants were randomly assigned to play the moral game $(51.7 \%)$, while 84 played the neutral game version $(48.3 \%)$. 


\subsection{Measures}

Prosocial attitude toward cultural outgroups was measured by using a single item, which asked participants how much admiration they felt toward someone of a different nationality, measured on a seven-point Likert scale (1: Not At All; 7: Extremely). The adjective was also used in Sweetman et al. [41] and Pettigrew and Meerten's [46] study.

Meaningfulness was measured using Oliver and Bartsch's [47] adapted Appreciation Scale. The measure contained three items, rated on a seven-point Likert scale (1: Strongly Disagree; 7: Strongly Agree). Sample statements included "I found the story of this game to be very meaningful." A high reliability score was obtained $(\alpha=.93)$.

Gameplay enjoyment was measured using a single item where participants were asked to rate their feelings toward the overall gameplay, rated on a scale from 0 to 100 (0: Negative; 100: Positive).

\section{Results}

Descriptive statistics of the main variables were generated, prior to further analyses (Table 1).

Table 1. Descriptive statistics

\begin{tabular}{|c|c|c|c|}
\hline Variable & $\boldsymbol{M}(\mathbf{S D})$ & Min & Max \\
\hline Pre-Admiration & $4.14(1.19)$ & 1.00 & 7.00 \\
\hline Post-Admiration & $4.15(1.57)$ & 1.00 & 7.00 \\
\hline $\begin{array}{c}\text { Admiration } \\
\text { Change } \\
\text { (Post - Pre) }\end{array}$ & $0.01(1.44)$ & -4.00 & 5.00 \\
\hline $\begin{array}{c}\text { Game Play } \\
\text { Experience }\end{array}$ & $\begin{array}{c}58.95 \\
(20.42)\end{array}$ & 0.00 & 100.00 \\
\hline Meaningfulness & $4.02(1.49)$ & 1.00 & 7.00 \\
\hline
\end{tabular}

A multiple regression analysis was conducted to examine the relationship between players' perceived meaningfulness and their admiration change toward immigrants (RQ2). Players' demographic variables, such as age and gender were entered into the first block as control variables, followed by the meaningfulness and gameplay experience scores in the second block.

The regression model that predicted players' admiration change was not statistically significant (Table 2), $R^{2}=0.01$, adjusted $R^{2}=-0.01, F(4,168)=$ $0.59, p=.67$. Meaningfulness $(\beta=0.02,95 \%$ CI [-
$0.15,0.19], p=.81)$ and perceived gameplay experience $(\beta=0.07,95 \%$ CI $[-0.01,0.02], p=.47)$ did not show significant weights on admiration change.

Table 2. Multiple regression model to predict players' admiration change toward cultural outgroups

\begin{tabular}{|c|c|c|c|c|}
\hline Variable & $\boldsymbol{B}$ & $S E B$ & $\boldsymbol{\beta}$ & $p$ \\
\hline Constant & -0.30 & 1.24 & & n.s. \\
\hline Age & -0.02 & 0.04 & -0.03 & n.s. \\
\hline Gender & 0.19 & 0.25 & 0.06 & n.s. \\
\hline Meaningfulness & 0.02 & 0.09 & 0.02 & n.s. \\
\hline $\begin{array}{l}\text { Gameplay } \\
\text { Experience }\end{array}$ & 0.01 & 0.01 & 0.07 & n.s. \\
\hline
\end{tabular}

significant

An independent-samples $t$-test was performed to examine if players in the moral condition experienced higher levels of meaningfulness toward the game, compared to those in the neutral condition (H1). The test indicated that the average meaningfulness rating in the moral condition $(M=4.60, S D=1.25)$ was significantly higher than the average meaningfulness rating in the neutral condition $(M=3.40, S D=1.48)$, $t(172)=5.77, p<.001$. The $95 \%$ confidence interval for the mean difference between the two conditions ranged from 0.79 to 1.60 . Therefore, $\mathrm{H} 1$ is supported.

In response to $\mathrm{H} 2$, an independent-samples $t$-test was conducted to understand if players in the moral condition experienced higher levels of game enjoyment than those in the neutral condition. The analysis showed that the mean gameplay rating in the moral condition $(M=63.49, S D=17.54)$ was significantly higher than the mean game play rating in the neutral condition $(M=54.08, S D=22.20), t(172)$ $=3.11, p=.00$. The $95 \%$ confidence interval for the mean difference between the two conditions ranged from 3.44 to 15.37 . Hence, $\mathrm{H} 2$ is also supported.

Similarly, an independent-samples $t$-test was conducted to find out if players in the moral condition reported higher levels of admiration change than those in the neutral condition (H3). The analysis showed that the mean admiration change score in the moral condition $(M=0.00, S D=1.32)$ was not significantly higher than the mean admiration change score in the neutral condition $(M=0.02, S D=1.57), t(172)=$ - 
$0.11, p=.91$. The $95 \%$ confidence interval for the mean difference between the two conditions ranged from -0.46 to 0.41 . The findings failed to support $\mathrm{H} 3$.

A multiple regression analysis was conducted among players in the moral condition to examine the association between meaningfulness and players' admiration change (H4). Demographic variables were entered in the first block, followed by meaningfulness and gameplay experience ratings in the next block.

Table 3. Multiple regression model to predict players' admiration change toward cultural outgroups in the moral condition

\begin{tabular}{|c|c|c|c|c|}
\hline Variable & $\boldsymbol{B}$ & $S E B$ & $\beta$ & $p$ \\
\hline Constant & -0.11 & 1.63 & & n.s. \\
\hline Age & -0.03 & 0.05 & -0.07 & n.s. \\
\hline Gender & 0.02 & 0.32 & 0.01 & n.s. \\
\hline Meaningfulness & 0.09 & 0.13 & 0.09 & n.s. \\
\hline $\begin{array}{l}\text { Gameplay } \\
\text { Experience }\end{array}$ & 0.01 & 0.01 & 0.07 & n.s. \\
\hline
\end{tabular}

significant

The regression model that predicted players' admiration change in the moral condition was not statistically significant (Table 3 ), $R^{2}=0.03$, adjusted $R^{2}=-0.02, F(4,85)=0.53, p=.71$. Meaningfulness $(\beta$ $=0.09,95 \% C I[-0.16,0.34], p=.47)$ and gameplay experience $(\beta=0.07,95 \%$ CI $[-0.01,0.02], p=.55)$ did not show any significant weights on admiration change. The findings failed to support $\mathrm{H} 4$.

\section{Discussion}

Overall, the game version that incorporated moral choices enabled players to experience higher levels of meaningfulness, relative to games that require players to make mundane choices (H1). This result aligns with Green and Jenkins' [29] argument that moral dilemmas in video games can evoke a sense of responsibility among players. In this purpose-made management game, players' choices in the moral scenarios could potentially influence their workers' wellbeing, such as decisions that could cause the workers to be injured. In contrast, players' decisions in the neutral game version were not described to have any significant impact on the workers. Therefore, players might have exerted more cognitive effort in the moral condition to decide if they should support their workers or the customers, which are both important stakeholders in ensuring smooth operations of the food establishment. These dilemmas might have led to a greater sense of perceived meaningfulness upon making those decisions.

Players enjoyed the meaningful game version with moral decisions more than those who played the less meaningful game version (H2). As the gameplay and controls in both games were designed similarly, only differing in moral content, this suggests that integrating both hedonic and eudaimonic experiences in a video game may be beneficial for improving overall enjoyment even in games that are meant to be more fun than meaningful. Although some scholars have suggested that fun games tend to be less cognitively demanding than meaningful ones [16], players could still subjectively decide whether they want to engage in the meaningful content and reflect on it, or to ignore it and focus on the less cognitively demanding aspects of the game. This can be observed in commercial games like Portal, for example, which provide both hedonic (puzzle-solving challenges) and eudaimonic (morally provoking story sequences) experiences for players, however, it is ultimately dependent on the player to decide how they want to play the game [27]. A player can choose to focus on the hedonic portion of the game and to ignore the eudaimonic portion of the game if they wish to. However, providing both types of enjoyment for players potentially covers more ground than focusing on only one aspect, particularly in serious games which have been criticized to focus too much on being meaningful but lack in fun compared to entertainment games [47]. In this specific game, players in the moral condition could also choose to avoid engaging in the moral decision-making (eudaimonic content) and to focus on maximizing profits in the simulation (hedonic content). However, these playstyle differences were not explored in the context of this study and may warrant further exploration to better understand how such playstyles may affect perceived meaningfulness and enjoyment. Additionally, although differences between perceived meaningfulness and game enjoyment were significant between the two conditions in the study, such findings may be context dependent and could possibly differ in different samples or with different situational contexts. Bartsch and Oliver [49], for example, suggest that meaningfulness and enjoyment in entertainment media could likely exist on an orthogonal scale, hence, although they may co-exist, this relationship and their corresponding intensity levels may vary.

We did not find any differences in admiration change toward cultural outgroups between the two 
versions of the game (H3 \& RQ2). This finding is similar to Tear and Nielsen's study [34], where the authors did not find any effects of playing prosocial video games and people's subsequent prosocial behavior, relative to those who played violent video games. It is possible that the degree of moral dilemma presented in the game has not reached the threshold to have a strong impact on players' attitudes, even though it is meaningful. Other gaming elements that were not studied in this paper, such as play-avatar identification, may have influenced the result. Future research can explore how other game design elements may also influence prosocial attitudes.

There was also no correlation found between players' perceived meaningfulness of the game and their subsequent admiration change toward outgroup members, failing to support H4. Although past literature suggested that contemplation about moral situations can relate to prosocial outcomes $[36,50]$, we did not find support for these effects in the current study. A combination of demand characteristics and an artificial ceiling effect could have contributed to this, as reported mean pre-admiration scores $(M=4.14, S D$ $=1.19$ ) were significantly higher than the midpoint score of 3.50 before the study began, $t(173)=7.08, p$ $<.001$, according to a one-sample $t$-test. Additionally, although the moral dilemmas may have contributed to the meaning-making process for players as investigated in $\mathrm{H} 1$, its link to the non-player characters involved in the interaction, and the duration of the scenarios may not have created sufficient contact between players and the related outgroup members. A pilot test of the scenarios playing out in the absence of other gameplay elements may have helped to better establish this link and finetune the design to better replicate parasocial contact.

The context of the eudaimonic design considerations may also benefit from further investigation. In this study, moral scenarios were designed based on real-life situations. Findings relating to narrative theory suggest that the use of fictional content may be equally as effective as nonfictional content, as it tends to be less value-laden by real-life knowledge and preconceptions, yet still be cognitively processed in relation to real-life situations $[51,52]$. Studies by Johnson [53] as well as Bal and Veltkamp [54] found that fiction reading was effective in eliciting empathy as well as influencing subsequent prosocial behavior. Similarly, in the context of video games, some anecdotal evidence can be observed in commercial games such as World of Warcraft, where players describe being faced with moral dilemmas within the fully fictional fantasy world $[55,56]$. Future studies could explore whether meaningful content (e.g., moral scenarios) based on fictional or non- fictional contexts within a video game may be more meaningful to players from more diverse backgrounds and provide additional insights and recommendations for designing effective meaningful experiences.

While this study found supporting evidence that the inclusion of moral decision-making in a video game can help to improve players' perceptions of meaningfulness (RQ1), it is also important to point out that the specific choices players had to make did not largely affect or delineate gameplay in any way. This meant that players were able to make a moral choice based on the interactive situation presented to them without worrying about consequential outcomes relating to gameplay (e.g., losing the game). This allowed players to navigate through various moral scenarios based on their own reflective thoughts and values, rather than acting in ways to secure their victory in the game. It is also important to note that while single-item measures for both participants' perceived gameplay experiences and admiration were used to reduce participants' fatigue after the video gameplay, they are limitations to this current study.

As the current study has focused on game dialogue choices, future research can investigate how other dimensions of gameplay may factor into or change players' moral decision-making processes, especially if it may influence winning or losing conditions in the game. Prior studies have found that for certain players, winning is a rewarding behavior and desired outcome of games, while losing can lead to an increase in negative affective states [57]. Hence, if moral choices are potentially tied to consequences that can cause a player to lose a game, they may be more inclined to choose the more desirable choices that can help them to win the game, rather than the choices that they subjectively believe in.

\section{Conclusion}

The current study contributed to the literature on meaningful play. It explains how eudaimonic (meaningfulness) and hedonic (enjoyment) gaming experiences coexist in our game. The difference in the gaming choices, designed based on morally loaded decisions vs. mundane conversational decisions have led to different degrees of meaningfulness and enjoyment. However, the potential links between meaningfulness, enjoyment, and serious outcomes of games warrants further exploration.

\section{Acknowledgments}

This research is supported by the Ministry of Education, Singapore, under its Academic Research Fund Tier 1 Grant (RG41/20). 


\section{References}

[1] K. Salen and E. Zimmerman, "Game design and meaningful play", In Handbook of Computer Game Studies, MIT Press, 2005, pp. 59-79.

[2] P. Sidhu and M. Carter, "Pivotal Play: Rethinking Meaningful Play in Games Through Death in Dungeons \& Dragons", Games and Culture, 2021, 15554120211005231.

[3] Y.J. Halbrook, A.T. O'Donnell, and R.M. Msetfi, "When and how video games can be good: A review of the positive effects of video games on well-being", Perspectives on Psychological Science, Vol. 14, No. 6, 2019, pp. 1096-1104.

[4] G.H. Iten, J.A. Bopp, C. Steiner, K. Opwis, and E.D. Mekler, "Does a prosocial decision in video games lead to increased prosocial real-life behavior? The impact of reward and reasoning", Computers in Human Behavior, Vol. 89, 2018, pp. 163-172.

[5] D.A. Gentile, C.A. Anderson, S. Yukawa, N. Ihori, M. Saleem, L.K. Ming, ... and A. Sakamoto, "The effects of prosocial video games on prosocial behaviors: International evidence from correlational, longitudinal, and experimental studies", Personality and Social Psychology Bulletin, Vol. 35, No. 6, 2009 , pp. $752-763$.

[6] Cambridge Dictionary, "Meaningfulness", Cambridge University Press, 2021.

[7] Merriam-Webster, "Meaningful", Merriam-Webster Incorporated, 2021.

[8] R.F. Baumeister and K.D. Vohs, "The pursuit of meaningfulness in life", In Handbook of Positive Psychology Vol. 1, Oxford University Press, 2002, pp. 608-618.

[9] F. Martela, R.M. Ryan, and M.F. Steiger, "Meaningfulness as satisfaction of autonomy, competence, relatedness, and beneficence: Comparing the four satisfactions and positive affect as predictors of meaning in life", Journal of Happiness Studies, Vol. 19, No. 5, 2018, pp. 12611282.

[10] T. Vuori, E. San, and M. Kira, "Meaningfulnessmaking at work", Qualitative research in organizations and management: An International Journal, Vol. 7, No. 2, 2012, pp. 231-248.

[11] S.A. Woods and J.A. Sofat, "Personality and engagement at work: The mediating role of psychological meaningfulness", Journal of Applied Social Psychology, Vol. 43, No. 11, pp. 2203-2210.

[12] S. Laato, S. Rauti, A.N. Islam, and E. Sutinen, "Why playing augmented reality games feels meaningful to players? The roles of imagination and social experience", Computers in Human Behavior, Vol. 121, 2021, 106816.

[13] C.A. Oswald, C. Prorock, and S.M. Murphy, "The perceived meaning of the video game experience: An exploratory study", Psychology of Popular Media Culture, Vol. 3, No. 2, 2014, pp. 110-126.

[14] A. Della Fave, F. Massimini, and M. Bassi, "Hedonism and eudaimonism in positive psychology", In Psychological Selection and Optimal
Experience Across Cultures, Springer, Dordrecht, 2011, pp. 3-18.

[15] M.B. Oliver, and J.K. Woolley, "Tragic and poignant entertainment: The gratifications of meaningfulness as emotional response", In The Routledge Handbook of Emotions and Mass Media, Routledge, 2010, pp. 148-161.

[16] M.B. Oliver and A. Bartsch, "Appreciation of entertainment", Journal of Media Psychology, Vol. 23, No. 1, 2011, pp. 29-33.

[17] N. Krämer, S.C. Eimler, G. Neubaum, S. Winter, L. Rösner, and M.B. Oliver, "Broadcasting one world: How watching online videos can elicit elevation and reduce stereotypes", New Media \& Society, Vol. 19, No. 9, 2016, pp. 1349-1368.

[18] F.M. Schneider, C. Weinmann, F.S. Roth, K. Knop, and P. Vorderer, "Learning from entertaining online video clips? Enjoyment and appreciation and their differential relationships with knowledge and behavioral intentions", Computers in Human Behavior, Vol. 54, 2016, pp. 475-482.

[19] S.T. Steinemann, E.D. Mekler, and K. Opwis, "Increasing donating behavior through a game for change: The role of interactivity and appreciation", In Proceedings of the 2015 Annual Symposium on Computer-Human Interaction in Play, 2015, pp. 319329.

[20] I. Bogost, "How to do things with videogames", U of Minnesota Press, 2011.

[21] S.B. Schafer, and G. Yu, "Meaningful video games: drama-based video games as transformational experience", In Business, Technological, and Social Dimensions of Computer Games: Multidisciplinary Developments, IGI Global, 2011, pp. 312-329.

[22] J.L. Nay and J.P. Zagal, "Meaning without consequence: virtue ethics and inconsequential choices in games", In Proceedings of the 12th International Conference on the Foundations of Digital Games, 2017, pp. 1-8.

[23] S. Stang, "This action will have consequences: Interactivity and player agency", Game Studies, Vol. 19, No. 1, 2019.

[24] M.B. Oliver, N.D. Bowman, J.K. Woolley, R. Rogers, B.I. Sherrick, and M.-Y. Chung, "Video games as meaningful entertainment experiences", Psychology of Popular Media Culture, Vol. 5, No. 4, 2016, pp. 390-405.

[25] B. Ip, "Narrative structures in computer and video games: Part 1: Context, definitions, and initial findings", Games and Culture, Vol. 6, No. 2, 2011, pp. 103-134.

[26] K.A. Bowen, "The realm of make believe and the extension of gameplay space", The International Journal of Creativity and Problem Solving, Vol. 25, No. 2, 2015, pp. 37-47.

[27] R. Rogers, J.K. Woolley, B.I. Sherrick, N.D. Bowman, and M.B. Oliver, "Fun versus meaningful video game experiences: A qualitative analysis of user responses", The Computer Games Journal, Vol 6. No. 1-2, 2017, pp. 63-79. 
[28] Ø. Kvalnes, (2019) "Moral Dilemmas. In: Moral Reasoning at Work”, Palgrave Pivot, Cham, 2019, pp. 11-19.

[29] M.C. Green, and K.M. Jenkins, "Interactive narratives: Processes and outcomes in user-directed stories", Journal of Communication, Vol. 64, No. 3, 2014, pp. 479-500.

[30] J. Graham, J. Haidt, S. Koleva, M. Motyl, R. Iyer, S.P. Wojcik, \& P.H. Ditto, "Moral foundations theory: The pragmatic validity of moral pluralism", Advances in Experimental Social Psychology, Vol. 47, 2013, pp. 55-130. Academic Press.

[31] J. Katsarov, M. Christen, R. Mauerhofer, D. Schmocker, and C. Tanner, "Training moral sensitivity through video games: A review of suitable game mechanisms", Games and Culture, Vol. 14, No. 4, 2019, pp. 344-366.

[32] L.O. Boltz, D. Henriksen, and P. Mishra, "Rethinking technology \& creativity in the 21 st century: Empathy through gaming-perspective taking in a complex world", TechTrends, Vol. 59, No. 6, 2015, pp. 3-8.

[33] A.S. Kümpel, and J. Unkel, "The effects of digital games on hedonic, eudaimonic and telic entertainment experiences.", Journal of Gaming \& Virtual Worlds, Vol. 9, No. 1, 2017, pp. 21-37.

[34] M. J. Tear and M. Nielsen. "Failure to demonstrate that playing violent video game diminishes prosocial behavior", PloS One, Vol. 8, No. 7, e68382

[35] R. Tamborini, M. Grizzard, N.D. Bowman, L. Reinecke, R.J. Lewis, and A. Eden, "Media enjoyment as need satisfaction: The contribution of hedonic and nonhedonic needs", Journal of Communication, Vol. 61, No. 6, 2011, pp. 10251042.

[36] S.T. Steinemann, G.H. Iten, K. Opwis, S.F. Forde, L. Frasseck, and E.D. Mekler, "Interactive narratives affecting social change", Journal of Media Psychology, Vol. 29, No. 1, 2017, pp. 54-66.

[37] E. Holl, S. Bernard, and A. Melzer, "Moral decisionmaking in video games: A focus group study on player perceptions", Human Behavior and Emerging Technologies, Vol. 2, No. 3, 2020, pp. 278-287.

[38] A. J. Weaver and N. Lewis. "Mirrored morality: An exploration of moral choice in video games", Cyberpsychology, Behavior, and Social Networking, Vol. 15, No. 11, 2012, pp. 610-614.

[39] T. Hartmann, E. Toz, and M. Brandon. "Just a Game? Unjustified Virtual Violence Produces Guilt in Empathetic Players", Media Psychology, Vol. 13, No. 4, 2010, pp. 339-363.

[40] A.A. Raney, "Disposition-based theories of enjoyment", Communication and Emotion, 2003, pp. 69-92.

[41] J. Sweetman, R. Spears, A. G. Livingstone, and A. S. Manstead. "Admiration regulates social hierarchy: Antecedents, dispositions, and effects of intergroup behavior", Journal of Experimental Social Psychology, Vol. 49, No. 3, 2013, pp. 534-542.

[42] I. Schindler, "Relations of admiration and adoration with other emotions and well-being", Psychology of Well-being, Vol. 4, No. 1, 2014, pp. 1-23.
[43] E. Schiappa, P.B. Gregg, D.E. Hewes, "The parasocial contact hypothesis", Communication Monographs, Vol. 72, No. 1, 2005, pp. 92-115.

[44] P. Breves, "Reducing outgroup bias through intergroup contact with non-playable video game characters in VR", Presence, Vol. 27, No. 3, 2020, pp. 257-273.

[45] J. Schell, "The art of game design: A book of lenses", CRC Press, 2008.

[46] T.F. Pettigrew, and R.W. Meertens, "Subtle and blatant prejudice in Western Europe", European Journal of Social Psychology, Vol. 25, No. 1, 1995, pp. 57-75.

[47] M.B. Oliver, A. Bartsch, "Appreciation as Audience Response: Exploring Entertainment Gratifications Beyond Hedonism", Human Communication Research, Vol. 36, No. 1, 2010, pp. 53-81.

[48] C. Shen, H. Wang, U. Ritterfeld, "Serious games and seriously fun games", In Serious games: Mechanisms and effects, Routledge, 2009, pp. 48-61.

[49] A. Bartsch, and M.B. Oliver, "Appreciation of meaningful entertainment experiences and eudaimonic well-being", In The Routledge handbook of media use and well-being: International perspectives on theory and research on positive media effects, Routledge, 2016, pp. 80-92.

[50] S.T. Steinemann, G.H. Iten, K. Opwis, S.F. Forde, L. Frasseck, and E.D. Mekler, "Interactive narratives affecting social change", Journal of Media Psychology, Vol. 29, No. 1, 2017, pp. 54-66.

[51] M. Appel, and T. Richter, "Transportation and need for affect in narrative persuasion: A mediated moderation model", Media Psychology, Vol 13., No. 2, 2010, pp. 101-135.

[52] M.A. Bekalu, C.A. Bigman, R.F. McCloud, L.K. Lin, and $\mathrm{K}$. Viswanath, "The relative persuasiveness of narrative versus non-narrative health messages in public health emergency communication: Evidence from a field experiment", Preventive Medicine, Vol. 111, 2018, pp. 284-290.

[53] D.R. Johnson, "Transportation into a story increases empathy, prosocial behavior, and perceptual bias toward fearful expressions", Personality and Individual Differences, Vol. 52, 2012, pp. 150-155.

[54] P.M. Bal, and M. Veltkamp, "How does fiction reading influence empathy? An experimental investigation on the role of emotional transportation", PloS One, Vol. 8, No. 1, 2013, e55341.

[55] T. Krzywinska, "Blood scythes, festivals, quests, and backstories: World creation and rhetorics of myth in World of Warcraft", Games and Culture, Vol. 1, No. 4, 2006, pp. 383-396.

[56] K. Gabriels, "Dealing with the virtual other: An empirical and ethical study on the un/acceptability of virtual moral practices", In Paper presented at The Power of Information Conference, Brussels, January 20-23, 2013.

[57] A. Dowsett, and M. Jackson, "The effect of violence and competition within video games on aggression", Computers in Human Behavior, Vol. 99, 2019, pp. 22-27. 